\title{
A parametrized version of the Borsuk-Ulam-Bourgin-Yang-Volovikov theorem
}

\author{
Benjamin Matschke*
}

September 27, 2013

\begin{abstract}
We present a parametrized version of Volovikov's powerful Borsuk-Ulam-BourginYang type theorem, based on a new Fadell-Husseini type ideal-valued index of G-bundles which makes computations easy.

As an application we provide a parametrized version of the following waist of the sphere theorem due to Gromov, Memarian, and Karasev-Volovikov: Any map $f$ from an $n$-sphere to a $k$-manifold $(n \geq k)$ has a preimage $f^{-1}(z)$ whose epsilon-neighborhoods are at least as large as the epsilon-neighborhoods of the equator $S^{n-k}$ (if $n=k$ we further need that $f$ has even degree).
\end{abstract}

\section{Introduction}

Volovikiv's theorem. Volovikov presented in [Vol92, Theorem 1] a strong Bourgin-Yang type theorem on point coincidences that also generalizes the Borsuk-Ulam theorem. The notation, in particular the index $\operatorname{ind}_{G}^{F H}(X)$ of a $G$-space, will be explained in section 3 .

Theorem 1.1 (Volovikov). Let $q=p^{k}$ be a prime power, $G=\mathbb{Z}_{p}^{k}$ the corresponding elementary Abelian group, and let $X$ be a free $G$-space of index $\operatorname{ind}_{G}^{F H}(X) \subseteq H^{* \geq m\left(p^{k}-1\right)+N}(G)$ with $N \geq 1$. Let $M$ be a compact m-manifold that is orientable if $p>2$. Suppose the $f^{*}: H^{*}(M) \rightarrow H^{*}(X)$ is zero for $i \geq 1$.

Then the set

$$
S:=\{x \in X|| f(G \cdot x) \mid=1\}
$$

is non-empty and has index $\operatorname{ind}_{G}^{F H} S \subseteq H^{* \geq N}(G)$.

For $k=1$, this theorem was already obtained in Volovikov [Vol80] and [Vol83]. Karasev and Volovikov KV11 generalized theorem 1.1 further to non-orientable manifolds and to arbitrary groups $\mathbb{Z}_{p}^{k} \subseteq G \subseteq \operatorname{Syl}_{p}\left(S_{q}\right)$.

The main methodological tool for this paper is a parametrized version of Volovikov's theorem, which we state in Section 4 .

Many other parametrized versions of the Borsuk-Ulam theorem are known. We refer to Jaworowski [Jaw81a], [Jaw81b] and [Jaw04], Dold [Dol88], Nakaoka [Nak84] and [Nak89], FadellHusseini [FH87a], [FH88] and [FH89], Živaljević-Vrećica [ŽV90], Izydorek-Jaworowski [IJ92],

${ }^{*}$ Supported by NSF Grant DMS-0635607 at IAS and by an EPDI fellowship at IHES and FIM (ETH Zürich). 
Izydorek-Rybicki [IR92, Mramor-Kosta [MK95], Volovikov [Vol96], Koikara-Mukerjee [KM96, Živaljević [Živ99], de Mattos-dos Santos dMdS07], Crabb-Jaworowski CJ09, Schick-SimonSpiecż-Toruńczyk [SSST11], Blagojević-M.-Ziegler [BMZ11] and [Mat11], and Singh [Sin11].

Main application. Gromov proved in [Gro03] the following version of the Borsuk-Ulam theorem.

Theorem 1.2 (Gromov's waist of the sphere theorem). Let $f: S^{n} \rightarrow \mathbb{R}^{k}$ be a continuous map where $n \geq k \geq 0$.

Then there exists a point $z \in \mathbb{R}^{k}$ such that for any $\varepsilon>0$,

$$
\operatorname{vol}_{n}\left(U_{\varepsilon}\left(f^{-1}(z)\right)\right) \geq \operatorname{vol}_{n}\left(U_{\varepsilon}\left(S^{n-k}\right)\right) \text {. }
$$

Here, $\operatorname{vol}_{n}$ denotes the standard measure on $S^{n}, U_{\varepsilon}(X)$ denotes the $\varepsilon$-neighborhood of a set $X \subseteq S^{n}$ with respect to the standard metric on $S^{n}$, and $S^{n-k}$ is the $(n-k)$-dimensional equator of $S^{n}$.

Memarian [Mem09 gave a more detailed proof of Gromov's theorem. Karasev and Volovikov [KV11] generalized it to maps $f: S^{n} \rightarrow M$ of even degree from the $n$-sphere to arbitrary $k$-manifolds $M$, see figure 1 .

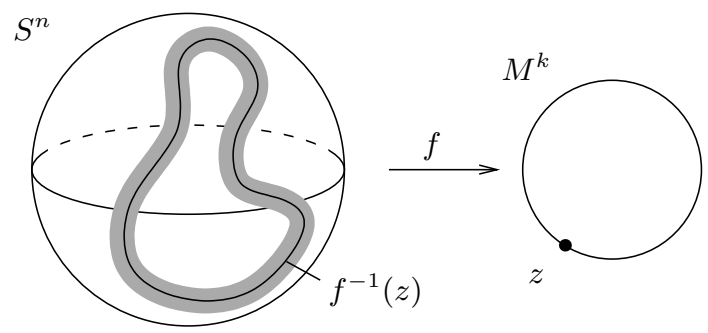

Figure 1: Example of the Gromov-Memarian-Karasev-Volovikov theorem for $n=2$ and $M=S^{1}$. In this example, $f^{-1}(z)$ is not a large preimage.

The main application of this paper is a parametrized version of this Gromov-MemarianKarasev-Volovikov theorem.

Theorem 1.3 (Parametrized Gromov-Memarian-Karasev-Volovikov waist of the sphere theorem). Let $f: B \times S^{n} \rightarrow E$ be a bundle map over $B$, where $S^{n} \hookrightarrow B \times S^{n} \rightarrow B$ is the trivial $S^{n}$ bundle over $B$ and $M \hookrightarrow E \stackrel{p}{\longrightarrow} B$ is a fiber bundle over $B$ whose fiber is a $k$-manifold $M$. If $n=k$ then we further assume that the fiber maps $f_{b}: S^{n} \rightarrow M$ have even degree at every base point $b \in B$.

Then there exist a subset $Z \subseteq E$ such that for all $z \in Z$ and $\varepsilon>0$,

$$
\operatorname{vol}_{n}\left(U_{\varepsilon}\left(f^{-1}(z)\right)\right) \geq \operatorname{vol}_{n}\left(U_{\varepsilon}\left(S^{n-k}\right)\right),
$$

and such that $Z$ is the set of limit points of a sequence of subsets $Z_{i} \subseteq E$ with

$$
\left(\left.p_{E}\right|_{Z}\right)^{*}: H^{*}\left(B ; \mathbb{F}_{2}\right) \rightarrow H^{*}\left(Z_{i} ; \mathbb{F}_{2}\right)
$$

being injective. Here, $\operatorname{vol}_{n}$ is the standard measure on the fiber $S^{n}$ over $p_{E}(z), U_{\varepsilon}($.$) denotes$ the $\varepsilon$-neighborhood in that fiber, and $H^{*}$ denotes Čech cohomology. 
Remark 1.4. It may happen the set $Z^{*}$ of all points $z \in E$ that satisfy the volume bound (1) for all $\varepsilon>0$ has the property that $H^{*}\left(B ; \mathbb{F}_{2}\right) \rightarrow H^{*}\left(Z^{*} ; \mathbb{F}_{2}\right)$ is not injective. For example this happens when $M=S^{n}$, the rank $n+1$ vector bundle associated to $p$ has non-trivial Stiefel-Whitney classes, and $f$ is wrapping enough to make $Z^{*}=E$.

The paper is organized as follows: We briefly discuss what we mean by parametrized theorems in section 2 (this section is rather philosophical and the reader may skip it without danger). In section 3 we define the index theories for $G$-bundles that are used in this paper and we discuss their basic properties. The parametrized Borsuk-Ulam-Bourgin-Yang-VolovikovKarasev theorem is stated in section 4 and it is proved in section 5. Finally, we prove the parametrized waist of the sphere theorem 1.3 in section 6 .

\section{Parametrized discrete geometry}

Many more theorems in discete geometry apart from Gromov's waist theorem have a parametrized version. A large class of such theorems are those that can be proved via what we would call the preimage method: For these theorems, the solution set can be described as a preimage $f^{-1}(Z)$ for some usually equivariant map $X \rightarrow Y \supset Z$, such that an invariant such as the equivariant bordism class of $f^{-1}(Z)$ does not vanish, which implies that the solution set is nonempty. This is a specialization of the Configuration Space/Test Map scheme, see Živaljević [Živ96], [Živ98].

Some theorems from discrete geometry turn out to admit "stronger" parametrizations than others. Let's make this precise. Consider a theorem $T$ that asserts for every valid input datum $d \in D$ the existence of a solution $s$ in the space of candidates of solutions $X$. Here, $D$ and $T$ are assumed to be topological spaces. There may be several natural choices for $D$ and especially for $X$.

Let us assume right away that $X$ is a fiber bundle over $D, p: X \rightarrow D$, and that the solution set $S(d)$ for $d \in D$ lies in the fiber over $d$. If $X$ does not naturally have such a structure, then simply replace it with the trivial bundle $\operatorname{pr}_{2}: X \times D \rightarrow D$. Thus $S: D \rightarrow 2^{X}$ is a set-valued section of $p$. In discrete geometry it is often upper hemicontinuous, i.e. its graph is closed.

The strongest form of a parametrization for theorem $T$ would be a section $s: D \rightarrow X$ such that $s(d) \in S(d)$ for all $d \in D$. This appears often when $T$ admits a constructive existence proof. Let's call this a solution selection map.

Convex solution sets. Slightly weaker parametrizations occur when there is set-valued function $S^{\prime}: D \rightarrow 2^{X}$ with $S^{\prime}(d) \subseteq S(d)$ such that $S^{\prime}(d)$ is convex, where here we require $p: X \rightarrow D$ to be a vector bundle.

The easiest example is probably Helly's theorem [Hel23] (see also Matoušek [Mat02]).

Theorem 2.1 (Helly). Any given family of convex sets $C_{i}, i \in I$ in $\mathbb{R}^{d}$ have a point in common if any $d+1$ of them do.

For an input datum $d=\left(C_{i}\right)_{i \in I}$ (topologized by the Hausdorff topology with respect to some finite metric on $\mathbb{R}^{d}$, and the product topology) the solution set $S(d)=\bigcap_{i} C_{i}$ is already convex. However there is no solution selection map, a counter-example is depicted in figure 2 . 


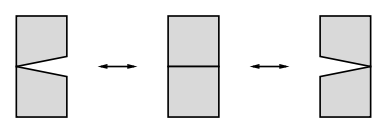

Figure 2: Example showing that there is no solution selection map for Helly's theorem.

A parametrized Helly theorem on vector bundles was proved and used by Dol'nikov [Dol87] and [Dol92] to establish the center transversal theorem, which is a generalization and interpolation between Banach's ham sandwich theorem and Rado's center point theorem.

Theorem 2.2 (Rado). Let $\mu$ be a probability measure on the Borel- $\sigma$-algebra of $\mathbb{R}^{d}$. Then there exists a point $c \in \mathbb{R}^{d}$, called the center point of $\mu$, such that any halfspace $H$ that contains $C$ satisfies $\mu(H) \geq 1 /(d+1)$.

Since the solutions set of all centerpoints of a given measure $\mu$ is convex, we get again immediately a parametrized version. This was first observed by Živaljević-Vrećica [ŽV90] who used it to prove the center transversal theorem, independently from Dol'nikov.

Weak parametrizations. Most often the there is not even a convex set valued solution selection function. Still there might be more quantitative assertions about the solution set $\mathbb{S}:=\cup_{d \in D} S(d)$, such as the injectivity of the map induced in some continuous cohomology $H^{*}(D) \rightarrow H^{*}(\mathbb{S})$, or the surjectivity of the map induced in some homology $H_{*}(\mathbb{S}) \rightarrow H_{*}(D)$.

A basic example is of course the Borsuk-Ulam theorem [Bor33.

Theorem 2.3 (Borsuk-Ulam). Any map $f: S^{d} \rightarrow \mathbb{R}^{d}$ sends some pair of antipodal points $x,-x \in S^{d}$ to the same point $f(x)=f(-x)$.

The solution set represents the generator of $H_{0}\left(\mathbb{R} P^{d} ; \mathbb{F}_{2}\right)=\mathbb{F}_{2}$, meaning that the generic number of solutions is odd. Jaworowski's parametrized version [Jaw81a concerns bundle maps from an $S^{d}$-bundle to a rank $d$ vector bundle $\varphi$ over the same base space $B$. He proved that if all Stiefel-Whitney classes up to $\omega_{i}$ of $\varphi$ are trivial, then $H^{d-i}\left(B ; \mathbb{F}_{2}\right) \rightarrow H^{d-i}\left(\mathbb{S} ; \mathbb{F}_{2}\right)$ is injective, $H^{*}$ being any continuous cohomology and $d$ being the cohomology dimension of $B$. For many more versions see the above references in the paragraph below remark 1.4 .

Another example is Tverberg's theorem [Tve66], [Tve81].

Theorem 2.4 (Tverberg). Let $N:=(r-1)(d+1)$. Any $N+1$ points in $\mathbb{R}^{d}$ can be partitioned into $r$ parts whose convex hulls have a point in common.

We could replace $\mathbb{R}^{d}$ by some rank $d$ vector bundle $\varphi$ over a base space $D$, and replace the given point set by $N+1$ sections in $\varphi$. The union $\mathbb{S}$ of the solution sets of Tverberg's theorem for every fiber of $\varphi$ will be over generic points $d \in D$ only a finite point set. But one can show that if $r=p^{k}$ is prime power then $\varphi$ induces an injection on Cech-cohomology $H^{*}\left(D ; \mathbb{F}_{p}\right) \rightarrow H^{*}\left(\mathbb{S} ; \mathbb{F}_{p}\right)$, see Živaljević [Živ99], Vrećica [Vre03, Karasev [Kar07], and Blagojević-M.-Ziegler [BMZ11]. These parametrized versions are then used to prove cases of the so-called transversal versions of Tverberg's theorem, the Tverberg-Vrećica conjecture [TV93]. More transversal versions of standard theorems in discrete geometry can be found in Karasev [Kar07, [Kar09b], and Montejano-Karasev [MK11] 


\section{Topological notations}

Let us fix some notation that will be used throughout the paper.

All spaces are paracompact, all maps are continuous. By a bundle we simply mean a map $X \rightarrow B$, where $X$ is called total space and $B$ base space. A $G$-bundle is a $G$-map $X \longrightarrow_{G} B$ from a $G$-space $X$ to a trivial $G$-space $B$. Base spaces will always be trivial $G$-spaces in this paper. In particular, $G$ acts fiberwise on $X$. When we write $F \hookrightarrow E \rightarrow B$ we mean a fiber bundle. Fiber bundles will always be locally trivial. A (Serre) $G$-fibration is a $G$-bundle with the $G$-equivariant lifting property for $G$-CW-complexes (usually $G$-fibrations are also defined for base spaces with non-trivial $G$-action, but not in this paper).

Let $q=p^{k}$ be a prime power. In this paper we consider only symmetry groups $G$ with $\mathbb{Z}_{p}^{k} \subseteq G \subseteq \operatorname{Syl}_{p}\left(S_{q}\right)$. Here, $S_{q}$ is the symmetric group on $q$ elements, $\mathbb{Z}_{p}=\mathbb{Z} /(p \mathbb{Z})$, and $\operatorname{Syl}_{p}\left(S_{q}\right)=\mathbb{Z}_{p} \imath \ldots \imath \mathbb{Z}_{p}$ is some $p$-Sylow subgroup of $S_{q}$. Cohomology groups $H^{*}(X)$ always denote Cech cohomology with $\mathbb{F}_{p}$-coefficients, which are constant coefficients except when we are talking about equivariant cohomology. In that case, the coefficients $\mathbb{F}_{p}$ are twisted by the sign of the permutation (remember that $G \subseteq S_{q}$ ).

For a $G$-space $X$, we write $X_{G}:=E G \times_{G} X$, which is the total space of the fibration $X \hookrightarrow X_{G} \rightarrow B$ called Borel construction. The $G$-equivariant cohomology (equivariant bundle cohomology, or Borel cohomology) of $X$ is $H_{G}^{*}(X):=H^{*}\left(X_{G}\right)$. In particular for a trivial $G$-space $B$ we have $H_{G}^{*}(B)=H^{*}\left(B_{G}\right)=H^{*}(G) \otimes H^{*}(B)$.

Let $W_{q}:=\left\{x \in \mathbb{R}^{q} \mid \sum x_{i}=0\right\}$ denote the standard representation of $S_{q}$. The $G$ equivariant Euler class of a $G$-bundle $F \hookrightarrow E \rightarrow B$ is the ordinary Euler class of the bundle $F \hookrightarrow E_{G} \rightarrow B_{G}$. The Euler class $e(V)$ of a $G$-representation $V$ is the equivariant Euler class of the bundle $V \hookrightarrow V \rightarrow$ pt, that is, the ordinary Euler class of $V \hookrightarrow V_{G} \rightarrow B G$.

For a vector bundle $F \hookrightarrow E \rightarrow B$ we denote the associated sphere and disk bundles by $S(F) \hookrightarrow S(E) \rightarrow B$ and $D(F) \hookrightarrow D(E) \rightarrow B$.

\subsection{Index theories}

In many situations one wants to disprove the existence of $G$-equivariant maps $X \longrightarrow_{G} Y \backslash Z$, or more generally that for some $G$-map $f: X \rightarrow Y \supset Z$ the preimage $f^{-1}(Z)$ is 'large' in some specific sense.

In our situation we are interested in $G$-bundle maps $f: X \rightarrow Y \supset Z$ over some trivial base space $B$. In this paper we will connect two different index theories, the first of which was defined and studied by Fadell and Husseini [FH87b], FH88].

Definition 3.1. Let $f: X \rightarrow B$ be a $G$-bundle. The Fadell-Husseini index of $f$ is defined as

$$
\operatorname{ind}_{B, G}^{F H}(X):=\operatorname{ker}\left(H_{G}^{*}(B) \stackrel{f^{*}}{\longrightarrow} H_{G}^{*}(X)\right) \subseteq H^{*}(G) \otimes H^{*}(B) .
$$

When $B$ is a point, we also write

$$
\operatorname{ind}_{G}^{F H}(X):=\operatorname{ind}_{\mathrm{pt}, G}^{F H}(X) \subseteq H^{*}(G) .
$$

Lemma 3.2 (Properties of $\operatorname{ind}_{B, G}^{F H}$ ). Let $f: X \longrightarrow_{G} B$ and $g: Y \longrightarrow_{G} B$ be $G$-maps with $B$ a trivial $G$-space as above. Then:

a) If there is a G-bundle map $h: X \longrightarrow_{G} Y$, that is $f=g \circ h$, then

$$
\operatorname{ind}_{B, G}^{F H}(X) \supseteq \operatorname{ind}_{B, G}^{F H}(Y) .
$$


b) If $X$ is n-connected then

$$
\operatorname{ind}_{G}^{F H}(X) \subseteq H^{* \geq n+2}(G) .
$$

c) If $F \hookrightarrow X \rightarrow B$ is a $G$-fibration and $F$ is $n$-connected, then

$$
\operatorname{ind}_{B, G}^{F H}(X) \subseteq H^{* \geq n+2}(B G \times B) .
$$

d) If $f: F \times B \rightarrow B$ is the projection to the second coordinate, then

$$
\operatorname{ind}_{B, G}^{F H}(F \times B)=\operatorname{ind}_{G}(F) \otimes H^{*}(B) .
$$

e) If $G=\mathbb{Z}_{p}^{k}$ then for any $G$-space $X$, $\operatorname{ind}_{G}^{F H}(X)=\emptyset$ if and only if $X$ has a fixed point.

f) If $\operatorname{ind}_{B, G}^{F H}(X) \cap H^{0}(G) \otimes H^{*}(B)=0$, then $f^{*}: H^{*}(B) \rightarrow H^{*}(X)$ is injective.

Proof. a) follows immediately from the definition and b) is the special case of c) for $F=X$ and $B=$ pt. c) follows from chasing the Leray-Serre spectral sequence of $F \hookrightarrow X_{G} \stackrel{f_{G}}{\longrightarrow}$ $B_{G}$ : Note that the index defining map $H_{G}^{*}(B) \stackrel{f^{*}}{\longrightarrow} H_{G}^{*}(X)$ coincides with the bottom edge homomorphism. Hence the elements of the index $\operatorname{ind}_{B, G}^{F H}(X)$ are exactly the elements in the bottom row of the spectral sequence that lie in the image of some differential. Since $F$ is $n$-connected, the only non-zero differentials hit the bottom row in filtration degree $n+2$ or higher.

d) follows from Künnet's theorem. For e), see tom Dieck [tD87, Prop. 3.14, p. 196]. f) follows immediately from the definition.

\subsection{A spectral sequence based index}

Let $f: X \rightarrow B$ be a $G$-bundle. If $f$ is not a $G$-fibration then we replace $X$ by $X^{\prime}:=$ $\{(x, \gamma) \mid x \in X, \gamma: I \rightarrow B, \gamma(0)=f(x)\}$ and $f$ by the map $f^{\prime}: X^{\prime} \rightarrow B$ that sends $(x, \gamma)$ to $\gamma(1)$. This replacement makes $f$ into a $G$-fibration, it is functorial, and if $f$ is a already a $G$-fibration then $f$ and $f^{\prime}$ are $G$-fiber homotopy equivalent. This gives several ways to define spectral-sequence based indices of $f$. For example, Blagojević-Blagojević-McCleary [BBM11] defined the spectral sequence witness of a pair of $G$-spaces $X$ and $X^{\prime}$ which gives a criterion for the non-existence of $G$-maps $X \rightarrow X^{\prime}$.

The index we will be interested in in this paper is the Leray-Serre spectral sequence of the map $X_{G}^{\prime} \rightarrow B$ given by $[e,(x, \gamma)] \mapsto f(\gamma(1))$. Here we need that $B$ is a trivial $G$-space. There is a natural map from $X_{G}^{\prime} \rightarrow B$ to $B_{G} \rightarrow B$, where $B_{G}=B G \times B$, which induces a morphism of associated spectral sequences.

Note that the spectral sequence of $B_{G} \rightarrow B$ collapes at $E_{2}^{*, *}=H^{*}(B) \otimes H^{*}(G)$. Also, any map bundle map $X \rightarrow Y$ over $B$ gives rise to a commutative triangle of maps between the associated spectral sequences.

In this paper it will be enough to consider the $E_{\infty}$-page.

Definition 3.3. Let $f: X \rightarrow B$ be a $G$-bundle. We define the $E_{\infty}$-index of $X \rightarrow B$ as

$$
\operatorname{ind}_{G, B}^{\infty}(X):=\operatorname{ker}\left(E_{\infty}^{*, *}\left(B_{G} \rightarrow B\right) \rightarrow E_{\infty}^{*, *}\left(X_{G}^{\prime} \rightarrow B\right)\right) \subseteq H^{*}(G) \otimes H^{*}(B) .
$$

By the multiplicativity of the Leray-Serre spectral sequence $\operatorname{ind}_{G, B}^{\infty}(X)$ is a bi-homogeneous ideal in $H^{*}(G) \otimes H^{*}(B)$. 
Lemma 3.4 (Properties of $\operatorname{ind}_{B, G}^{\infty}$ ). Let $X \rightarrow B$ be a G-bundle.

a) Let $X \rightarrow Y$ be a map of $G$-bundles over $B$. Then

$$
\operatorname{ind}_{B, G}^{\infty}(X) \supseteq \operatorname{ind}_{B, G}^{\infty}(Y) .
$$

b) If $F \times B \rightarrow B$ is the projection to the second coordinate then

$$
\operatorname{ind}_{B, G}^{\infty}(F \times B)=\operatorname{ind}_{\mathrm{pt}, G}^{F H}(F) \otimes H^{*}(B)=\operatorname{ind}_{B, G}^{F H}(F \times B) .
$$

c) If $\operatorname{ind}_{B, G}^{\infty}(X) \subseteq H^{* \geq 1}(G) \otimes H^{*}(B)$, then $f^{*}: H^{*}(B) \rightarrow H^{*}(X)$ is injective. If moreover $B$ is a compact manifold, then the map in singular homology $f_{*}: H_{*}\left(U_{\varepsilon}(X)\right) \rightarrow H_{*}(B)$ is surjective for any $\varepsilon>0$.

Proof. a) is a trivial chase in the diagram of the index defining spectral sequences. b) is trivial. c) follows from the definition and the edge-homomorphism.

Comparing ind ${ }^{F H}$ and ind ${ }^{\infty}$. Although both indices ind ${ }^{F H}$ and ind ${ }^{\infty}$ are similarly defined, there are substantial differences: First of all, ind ${ }^{\infty}$ is a $\mathbb{Z}^{2}$-graded ideal of $H^{*}(G) \otimes H^{*}(B)$, whereas ind ${ }^{F H}$ is only a $\mathbb{Z}$-graded ideal (with respect to the total grading).

Definition 3.5. We define the leading term $\operatorname{lt}(\alpha)$ of a homogeneous element $\alpha \in H^{*}(G) \otimes$ $H^{*}(B)$ as the first non-zero $\alpha_{i}$, where $\alpha=\alpha_{0}+\alpha_{1}+\ldots$ with $\alpha_{i} \in H^{*}(G) \otimes H^{i}(B)$. This extends degree-wise (with respect to the total degree) to a maps of sets lt: $H^{*}(G) \otimes H^{*}(B) \rightarrow$ $H^{*}(G) \otimes H^{*}(B)$.

Lemma 3.6. Any $G$-bundle $X \rightarrow B$ satisfies $\operatorname{lt}\left(\operatorname{ind}_{B, G}^{F H}(X)\right) \subseteq \operatorname{ind}_{B, G}^{\infty}(X)$.

However, the non-leading bihomogeneous parts of $\alpha \in \operatorname{ind}_{B, G}^{F H}(X)$ may not be in $\operatorname{ind}_{B, G}^{\infty}(X)$. Also bihomogeneous elements $\beta \in \operatorname{ind}_{B, G}^{\infty}(X)$ may not lie in $\operatorname{ind}_{B, G}^{F H}(X)$, because $\beta \in \operatorname{ind}_{B, G}^{\infty}(X)$ means that its image is zero in some filtration quotient of $H_{G}^{*}(X)$.

Example 3.7. As an example, let $p: X \rightarrow B$ be the associated circle bundle of the tangent bundle of $\mathbb{R} P^{2}$. Suppose that $G=\mathbb{Z}_{2}$ acts antipodally on each fiber of $X$. Then, $H^{*}(G)=\mathbb{F}_{2}[t]$ and $H^{*}(B)=\mathbb{F}_{2}[u] /\left(u^{3}\right)$. The associated fibration of Borel constructions, $X_{\mathbb{Z}_{2}} \rightarrow B G \times B$, is a circle bundle with $(\bmod 2)$ Euler class $e=u^{2}+t^{2}$. This is the generator for $\operatorname{ind}_{B, G}^{F H}(X)$. We have $\operatorname{lt}(e)=t^{2} \in \operatorname{ind}_{B, G}^{\infty}(X)$, and in fact $t^{2}$ is the generator of $\operatorname{ind}_{B, G}^{\infty}(X)$. Hence in this example, the other bihomogeneous part $u^{2}$ of $e \in \operatorname{ind}_{B, G}^{F H}(X)$ does not lie in $\operatorname{ind}_{B, G}^{\infty}(X)$, and the bihomogeneous element $t^{2} \in \operatorname{ind}_{B, G}^{\infty}(X)$ does not lie in $\operatorname{ind}_{B, G}^{F H}(X)$.

\subsubsection{More general versions}

The following versions and generalizations of ind ${ }^{\infty}$ may be useful for different problems, but we won't need them in this paper.

We can define to any $G$-bundle $f: X \rightarrow B$ the spectral sequence valued index $\operatorname{ind}_{G, B}^{S S}(X)$ as the Leray-Serre spectral sequence of $X_{G}^{\prime} \rightarrow B$ together with the morphism of spectral sequences from the spectral sequence of $B_{G} \rightarrow B$. In abstract terms this index is a functor from the category of $G$-bundles over $B$ to the category whose objects are morphisms from one spectral sequence to the spectral sequence of $B_{G} \rightarrow B$. 
Two other useful indices can be defined in a similar way using the fibrations $X \hookrightarrow X_{G} \rightarrow$ $B G$ and $F \hookrightarrow X_{G}^{\prime} \rightarrow B_{G}$, where $F$ is the homotopy fiber of $X \rightarrow B$. If $B$ is a point then both of them coincide. The latter contains all information of the Fadell-Husseini index, since $\operatorname{ind}_{G, B}(X)$ is the set of all elements in the 0-row of the spectral sequence of $X_{G}^{\prime} \rightarrow B_{G}$ that are in the image of some differential.

In some sense these three spectral sequence valued indices can be unified using a "higher spectral sequence" that will be constructed in [Mat12]. The corresponding sequence of two fibrations is $X_{G} \rightarrow B_{G} \rightarrow B$.

\section{Parametrized Volovikov theorem}

The main methodological tool in this paper is the following parametrized Volovikov theorem. It relates the $\infty$-index of the configuration space $X$ to the Fadell-Husseini index of the solution set $S$.

Theorem 4.1 (Parametrized Volovikov theorem). Let

- $q=p^{k}$,

- $G$ be a subgroup of $S_{q}$ such that $\mathbb{Z}_{p}^{k} \subseteq G \subseteq \operatorname{Syl}_{p}\left(S_{q}\right)$,

- B be a path-connected trivial G-space,

- $p_{X}: E_{X} \rightarrow B$ be a G-bundle,

- $Y$ be a paracompact space and let $G$ act on $Y^{q}$ by permuting the coordinates,

- $Y \hookrightarrow E_{Y} \rightarrow B$ be a fiber bundle,

- $M$ be a connected (paracompact) smooth m-manifold,

- $M \hookrightarrow E_{M} \rightarrow B$ be a fiber bundle.

Let $i: E_{X} \longrightarrow_{G} E_{Y}^{\oplus q}$ be a G-bundle map over $B$,

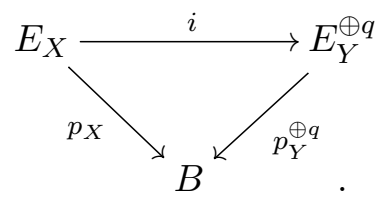

Let $F$ be a fiber bundle map,

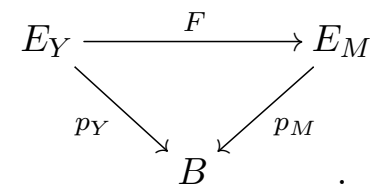

Assume that over some (and hence any) base point $b \in B$, the map $F_{b}:=\left.F\right|_{p_{Y}^{-1}(b)}=\left.F\right|_{Y}$ induces the zero map in positive cohomology $H^{* \geq 1}\left(M, \mathbb{F}_{p}\right) \rightarrow H^{* \geq 1}\left(Y, \mathbb{F}_{p}\right)$.

Then the "solution set"

$$
S:=\left\{x \in E_{X} \mid i(x)=\left(y_{1}, \ldots, y_{q}\right) \text { satisfies } F\left(y_{1}\right)=\ldots=F\left(y_{q}\right)\right\}
$$


has the following index bound: If $\alpha \in \operatorname{ind}_{B, G}^{F H}(S)$ is a homogeneous element then

$$
\operatorname{lt}(\alpha) e\left(W_{q}\right)^{m} \in \operatorname{ind}_{B, G}^{\infty}\left(E_{X}\right) .
$$

In particular if $\operatorname{ind}_{B, G}^{\infty}\left(E_{X}\right) \cap\left(e\left(W_{q}\right)^{n} \otimes H^{*}(B)\right)=\emptyset$ then

$$
H^{*}\left(B, \mathbb{F}_{p}\right) \stackrel{\left(\left.p_{M}\right|_{Z}\right)^{*}}{\longrightarrow} H^{*}\left(Z, \mathbb{F}_{p}\right)
$$

is injective, where

$$
\begin{aligned}
Z:=F^{q}(i(S)) \cong\left\{z \in E_{M} \mid\right. & z=F\left(y_{1}\right)=\ldots=F\left(y_{q}\right) \\
& \left.\quad \text { for some } x \in E_{X},\left(y_{1}, \ldots, y_{q}\right)=i(x)\right\} .
\end{aligned}
$$

Remark 4.2. In applications of theorem 4.1, $E_{X}$ is usually the configuration space (the space of solution candidates), which is parametrized over $B$, and $M$ is also naturally given by our description of the solution set as a preimage. But what about $Y \hookrightarrow E_{Y} \rightarrow B$ ? The assumption of the theorem that $F^{\oplus q} \circ i$ has to factor over $E_{Y}$ is important for rather technical reasons. Two cases for the choice of $E_{Y}$ usually appear:

1. $i: E_{X} \longrightarrow_{G} E_{Y}^{\oplus q}$ is an inclusion of $G$-spaces.

2. If $G=\mathbb{Z}_{p}^{k}$ and $E_{X} \rightarrow B$ is a fiber bundle, then one can simply choose $E_{Y}:=E_{X}$ and let $i: E_{X} \rightarrow E_{Y}^{\oplus q}$ be defined as $x \mapsto\left(g^{-1} x\right)_{g \in G}$.

Remark 4.3. The set $Z \subseteq E_{M}$ is in general much more complicated than the image of a section of $p_{M}: E_{M} \rightarrow B$ ( $p_{M}$ may not even admit a section).

Remark 4.4 (Desirable extensions). It would be useful to have a version of theorem 4.1 that relates $E_{X}$ and $S$ using the same index theory, such that one can apply the theorem iteratively.

If the "parametrized Nakaoka lemma" $H_{G}^{*}\left(E_{M}^{\oplus q}\right) \cong H^{*}\left(G ; H^{*}\left(E_{M}^{\oplus q}\right)\right.$ is true (and if this isomorphism is natural in $\left.p_{M}\right)$ then we would have the following relation: There exists $e^{\prime} \in$ $H_{G}^{m(q-1)}(B)$ with $\operatorname{lt}\left(e^{\prime}\right)=e\left(W_{q}\right)^{m} \otimes 1$ and $e^{\prime} \cdot \operatorname{ind}_{B, G}^{F H}(S) \subseteq \operatorname{ind}_{B, G}^{F H}\left(E_{X}\right)$.

\section{Proof of the parametrized Volovikov theorem}

In large parts we follow the proof of Volovikov [Vol92] (see $\S 5$ and in particular the proof of lemma 3) and Karasev-Volovikov [KV11].

We denote the $q$-fold Withney sum of $E_{M}$ by $M^{q} \hookrightarrow E_{M}^{\oplus q} \rightarrow B$. Let $\Delta_{M^{q}}$ denote the thin diagonal $\left\{(m, \ldots, m) \in M^{q}\right\}$ of $M^{q}$. Similarly, let $\Delta_{M^{q}} \hookrightarrow \Delta_{E_{M}^{\oplus q}} \rightarrow B$ be the thin diagonal subbundle of $E_{M}^{\oplus q}$.

$G \subseteq S_{q}$ acts on $M^{q}$ and $E_{M}^{\oplus q}$ by permuting coordinates. Their fixed-point sets are $\Delta_{M^{q}} \cong$ $M$ and $\Delta_{E_{M}^{\oplus q}} \cong E_{M}$, respectively.

Some closed tubular neighborhood $N\left(\Delta_{E_{M}^{\oplus q}}\right) \subset E_{M}^{\oplus q}$ can be regarded as a disc bundle $D^{m(q-1)} \hookrightarrow N\left(\Delta_{E_{M}^{\oplus q}}\right) \rightarrow \Delta_{E_{M}^{\oplus q}} \cong E_{m}$ of some $G$-vector bundle $W_{q}^{\oplus m} \hookrightarrow W \stackrel{\varphi}{\longrightarrow} \Delta_{E_{M}^{\oplus q}}$, the normal bundle of $\Delta_{E_{M}^{\oplus q}}$ in $E_{M}^{\oplus q}$. 
Let $\tau$ be the rank $m$ vector bundle over $\Delta_{E_{M}^{\oplus q}}$ whose fiber at a point $e \in \Delta_{E_{M}^{\oplus q}}$ is the tangent space $T_{e} M_{b}$, where $M_{b} \cong M$ is the fiber of $\Delta_{E_{M}^{\oplus q}} \rightarrow B$ that contains $e$. Then $\varphi \oplus \tau=\tau^{\oplus q}$, and $\varphi$ is stably equivalent to $\tau^{\oplus(q-1)}$. For $p>2, q-1$ is even. Hence $\varphi$ is $\mathbb{F}_{p}$-orientable. Furthermore all non-zero elements of $G$ have an odd order if $p>2$, thus the $G$-action preserves the $\mathbb{F}_{p}$-orientation on $\varphi$.

Therefore $W$ has a $G$-equivariant mod- $p$ Thom class $\tau_{E_{M}, G} \in H_{G}^{m(q-1)}(D(W), S(W))$, which is the ordinary mod-p Thom class of the bundle $W_{q}^{m} \hookrightarrow W_{G} \rightarrow\left(\Delta_{E_{M} q}\right)_{G}$, where $\left(\Delta_{E_{M}^{\oplus q}}\right)_{G}=\Delta_{E_{M}^{\oplus q}} \times B G$.

By excision we regard $\tau_{E_{M}, G}$ as an element in $H_{G}^{m(q-1)}\left(E_{M}^{\oplus q}, E_{M}^{\oplus q} \backslash \Delta_{E_{M}^{\oplus q}}\right)$. By the Thom isomorphism this group is isomorphic to $H_{G}^{0}\left(\Delta_{E_{M}^{\oplus q}}\right)=\mathbb{F}_{p}$.

Consider the diagram of restrictions

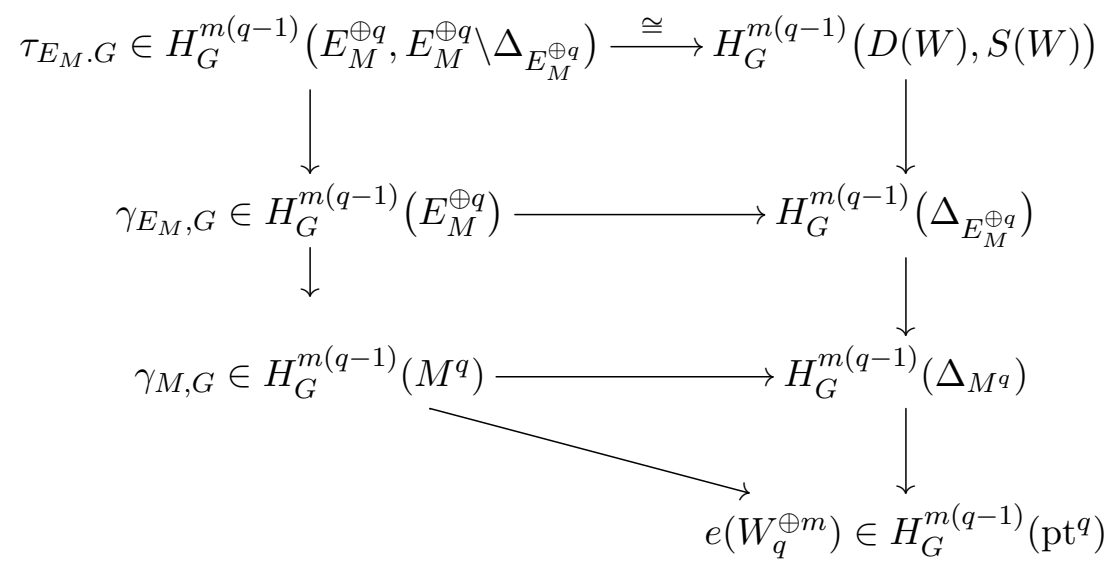

Let $\gamma_{E_{M}, G}$ denote image of $\tau_{E_{M}, G}$ in $H_{G}^{m(q-1)}\left(E_{M}^{\oplus q}\right)$. By commutativity of the top square, $\gamma_{E_{M}, G}$ maps to the Euler class $e(\varphi) \in H_{G}^{m(q-1)}\left(\Delta_{E_{M}^{\oplus q}}\right)$ of $\varphi$. Thus, when further restricting to $H_{G}^{m(q-1)}\left(\mathrm{pt}^{q}\right), \mathrm{pt}^{q}$ being some point in $\Delta_{E_{M}^{\oplus q}}, \gamma_{E_{M}, G}$ maps to $e\left(W_{q}\right)^{m}$.

Remark 5.1. In case $B$ is a manifold, $\tau_{E_{M}, G}$ can be constructed as the Poincaré dual of $E_{r} G \times{ }_{G} \Delta_{E_{M}^{\oplus q}}$ in $E_{r} G \times{ }_{G} E_{M}^{\oplus q}$, where $E_{r} G$ is an $r$-connected free $G$-manifold and $r \geq m(q-1)$, using the canonical isomorphism

$$
H^{m(q-1)}\left(E_{r} G \times_{G}\left(E_{M}^{\oplus q}, E_{M}^{\oplus q} \backslash \Delta_{E_{M}^{\oplus q}}\right)\right) \stackrel{\cong}{\longrightarrow} H_{G}^{m(q-1)}\left(E_{M}^{\oplus q}, E_{M}^{\oplus q} \backslash \Delta_{E_{M}^{\oplus q}}\right) .
$$

See Volovikov [Vol92] and Karasev-Volovikov [KV11] for details in the case when $B=$ pt.

$$
\text { Let } \gamma_{E_{X}}:=\left(F^{q} \circ i\right)^{*}\left(\gamma_{E_{M}, G}\right) \in H_{G}^{m(q-1)}\left(E_{X}\right) \text {. }
$$

Claim 5.2. The restiction of $\gamma_{E_{X}}$ to $E_{X} \backslash S$ is zero in $H_{G}^{m(q-1)}\left(E_{X} \backslash S\right)$.

Proof. From the long exact sequence of the pair $\left(E_{M}^{\oplus q}, E_{M}^{\oplus q} \backslash \Delta_{E_{M}^{\oplus q}}\right)$ we see that $\gamma_{E_{M}, G}$ restricts to zero in $H_{G}^{m(q-1)}\left(E_{M}^{\oplus q} \backslash \Delta_{E_{M}^{\oplus q}}\right)$. Since $F^{q} \circ i$ sends the pair $\left(E_{X}, S\right)$ to $\left(E_{M}^{\oplus q}, \Delta_{E_{M}^{\oplus q}}\right)$, the claim follows. 


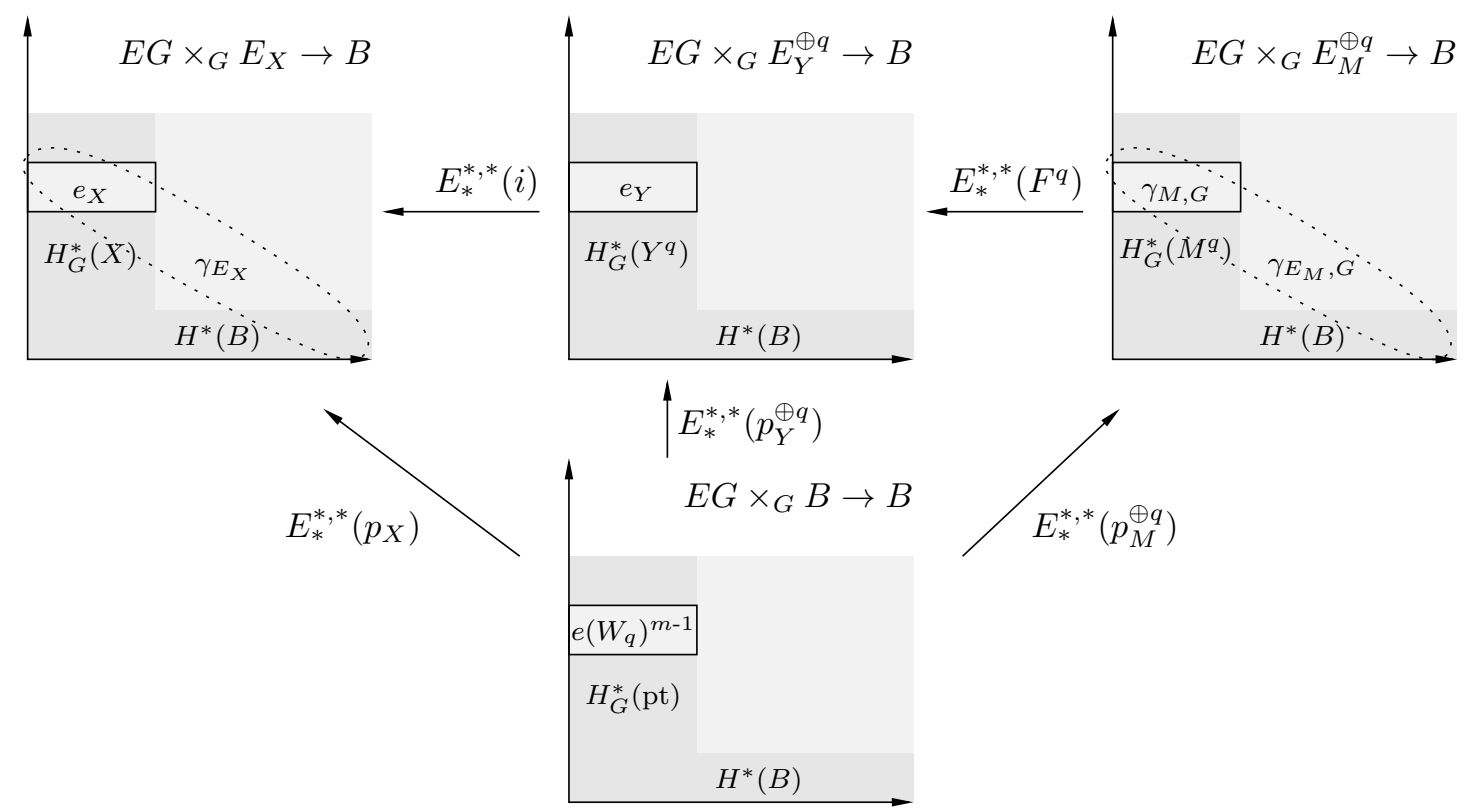

Figure 3: Seconds pages of the spectral sequences of $\left(E_{X}\right)_{G},\left(E_{Y}^{\oplus q}\right)_{G},\left(E_{M}^{\oplus q}\right)_{G}$, and $B_{G}$ over $B$.

The constructions of $\tau_{E_{M}, G}$ and $\gamma_{E_{M}, G}$ are natural with respect to taking subgroups of $G$ and restrictions of $B$. When restricting $E_{M}$ to a fiber $M_{b} \cong M$ over some base point $b \in B$, $\gamma_{E_{M}, G}$ restricts to an element $\gamma_{M, G} \in H_{G}^{m(q-1)}\left(M^{q}\right)$.

Now consider the diagram of spectral sequences in figure 3 . Here, $X$ is the homotopy fiber of $p_{X}: E_{X} \rightarrow B$, and $e_{X} \in H_{G}^{*}(X)$ and $e_{Y} \in H_{G}^{*}\left(Y^{\oplus q}\right)$ are the natural images of $e\left(W_{q}\right)^{m-1}$.

Claim 5.3. $E_{2}^{0, m(q-1)}\left(F^{q}\right)$ sends $\gamma_{M, G}$ to $e_{Y}$.

Proof. By Nakaoka's lemma [Nak61], we have an isomorphism

$$
H_{G}^{*}\left(M^{q}\right)=\operatorname{Tot}\left(H^{*}\left(G ; H^{*}(M)^{\otimes q}\right)\right),
$$

which is natural in $G$ and $M$, where Tot denotes the total complex of a bigraded complex. As an $\mathbb{F}_{p}[G]$-algebra, $H^{*}(M)^{\otimes q}$ decomposes as $\mathcal{A}+\mathcal{B}$, where $\mathcal{A}=H^{0}(M)^{\otimes q}=\mathbb{F}_{p}$ and $\mathcal{B}$ is generated by all homogeneous elements in $H^{*}(M)^{\otimes q}$ of positive total degree. Hence $\gamma_{M, G}$ decomposes as $\gamma_{M, G}=\gamma_{a}+\gamma_{b}$, where $\gamma_{a} \in H^{*}(G ; \mathcal{A})=H^{*}(G)$ and $\gamma_{b} \in H^{*}(G ; \mathcal{B})$.

The composition

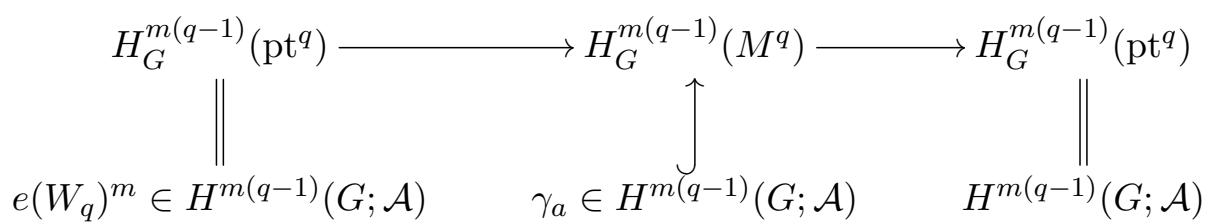

induced by the projection on the left and by an inclusion on the right is the identity. Both maps are also individually isomorphisms on the $H^{m(q-1)}(G ; \mathcal{A})$-part since the Nakaoka lemma is natural in $M$. Since the second map sends $\gamma_{M, G}$ to $e\left(W_{q}\right)^{m}$ we deduce that $\gamma_{a}=e\left(W_{q}\right)^{m}$ and the first map sends $e\left(W_{q}\right)^{m}$ to $\gamma_{a}$. Thus $E_{2}^{0, m(q-1)}\left(F^{q}\right)$ sends $\gamma_{a}$ to $e_{Y}$. 
$F: E_{Y} \rightarrow E_{M}$ restricts over some base point $b \in B$ to $F_{b}: Y^{q} \rightarrow M^{q}$, which by assumption induces zero in positive cohomology. Therefore $E_{2}^{0, m(q-1)}\left(F^{q}\right)$ will send $\gamma_{b}$ to zero, by naturality of Nakaoka's lemma.

From the claim follows that $E_{2}^{0, m(q-1)}\left(F^{q} \circ i\right)$ sends $\gamma_{M, G}$ to $e_{X}$. Since $\left(F^{q} \circ i\right)^{*}\left(\gamma_{E_{M}, G}\right)=$ $\gamma_{E_{X}}$ and $\gamma_{M, G}$ is the restriction of $\gamma_{E_{M}, G}$ to the first column of the right spectral sequence, $e_{X}$ must be the restriction of $\gamma_{E_{X}}$ on the left spectral sequence. In other words, $e_{X}$ is the leading term of $\gamma_{E_{X}}$ in the left spectral sequence.

Now suppose we are given a homogeneous element $\alpha \in \operatorname{ind}_{B, G}^{F H}(S)$, that is, $\alpha$ maps to zero in $H_{G}^{*}(S)$. By claim 5.2 if follows that

$$
\left(p_{X}\right)^{*}(\alpha) \cup \gamma_{E_{X}}=0
$$

Thus on the $E_{\infty}$-page we have that

$$
E_{\infty}^{0, m(q-1)}\left(p_{X}\right)\left(\operatorname{lt}(\alpha) \cup e\left(W_{q}\right)^{m}\right)=0 .
$$

This proves the general index bound for $S$.

For the last part of the theorem, assume that $\operatorname{ind}_{B, G}^{\infty}\left(E_{X}\right) \subseteq H^{* \geq m\left(p^{k}-1\right)+1}(G) \otimes H^{*}(B)$. Then the index bound yields that ind ${ }_{B, G}^{F H}(S)$ cannot contain elements in $H^{0}(G) \otimes H^{*}(B)$ except for 0 . Therefore lemma 3.4 implies that $H^{*}(B)=H^{0}(G) \otimes H^{*}(B) \rightarrow H_{G}^{*}(S)$ is injective. The commutative diagram of natural maps

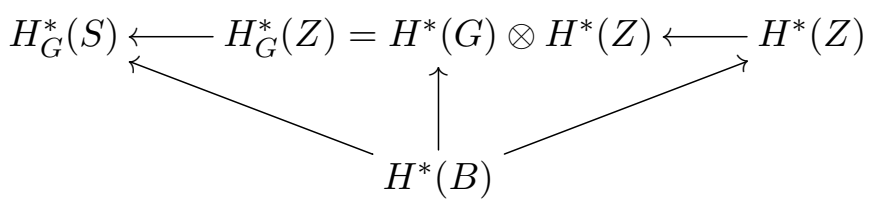

implies that $H^{*}(B) \rightarrow H^{*}(Z)$ is injective as well. This finishes the proof of theorem 4.1 .

\section{Sketch of proof of the parametrized waist of sphere theorem}

In the case $n=k$, the parametrized waist of the sphere theorem 1.3 follows easily from a parametrized Borsuk-Ulam theorem for manifolds: Theorem 4.1 implies for the given bundle map $B \times S^{n} \rightarrow E$ and the antipodal $\mathbb{Z}_{2}$-action on the fibers of $B \times S^{n}$ that the set $Z$ of all elements $z \in E_{M}$ whose preimage $f^{-1}(z)$ contains a pair of antipodal points has the property that $H^{*}\left(B ; \mathbb{F}_{2}\right) \rightarrow H^{*}\left(Z ; \mathbb{F}_{2}\right)$ is injective.

Thus we may assume $n>k$. Gromov's proof of 1.2 splits into a topological and an analytic part. The topological part is the following mass partition theorem.

Let $\operatorname{Conv}\left(S^{n}\right)$ denote the set of all closed convex subsets of $C \subset S^{n}$ with $C \neq S^{n}$. Let $\operatorname{Conv}^{*}\left(S^{n}\right)$ be its subset of sets with positive volume. The Hausdorff metic makes $\operatorname{Conv}\left(S^{n}\right)$ into a metric space. A map $c: \operatorname{Conv}^{*}\left(S^{n}\right) \rightarrow S^{n}$ is called a center map. A partition of $S^{n}$ into $q$ convex sets is a family of subsets $C_{1}, \ldots, C_{q} \in \operatorname{Conv}\left(S^{n}\right)$ with pairwise disjoint interior such that $S^{n}=\bigcup_{i} C_{i}$. 
Theorem 6.1 (A mass partition theorem). Let $g: S^{n} \rightarrow M^{k}$ be map from the $n$-sphere to a $k$-manifold, $n>k$, let $c: \operatorname{Conv}^{*}(S) \rightarrow S^{n}$ be a center map. Then for any $q=2^{\ell}$ there exists a partition of $S^{n}$ into $q$ convex sets $C_{1}, \ldots, C_{q}$ with

$$
g\left(c\left(C_{1}\right)\right)=\ldots=g\left(c\left(C_{q}\right)\right)
$$

and

$$
\operatorname{vol}\left(C_{1}\right)=\ldots=\operatorname{vol}\left(C_{q}\right) .
$$

Moreover the set $C_{i}$ can be required to lie in the $\varepsilon$-neighborhood of some $k$-dimensional equator $E_{i} \subset S^{n}$ in case $q \geq q_{0}(\varepsilon)$.

The analytic part of the proof is based on involved isoperimetric inequalities that make theorem 6.1 with $\varepsilon \rightarrow 0$ imply theorem 1.2, see Gromov [Gro03], Memarian [Mem09].

Every point $x \in S^{n}$ determines its polar hyperplane, which bisects $S^{n}$ into two convex pieces. Two more points on the sphere, one for each of the two pieces, will yield a convex partition of $S^{n}$ into four pieces. Iterating this, we obtain a map

$$
p: X:=\left(S^{n}\right)^{q-1} \rightarrow \operatorname{Conv}\left(S^{n}\right)^{q} .
$$

Let $T$ be the complete binary tree of height $\ell-1$. The interior nodes of $T$ naturally correspond to the $q-1$ sphere factors of $X$, and the $q$ leaves correspond to the convex sets in the partition. Let them be labelled by $N_{1}, \ldots, N_{q-1}$, where $N_{1}$ shall denote the root. Let the leaves of $T$ be labelled by $L_{1}, \ldots, L_{q}$. Thus the symmetry group of $T$, the 2 -Sylow subgroup $G:=\mathbb{Z}_{2} \imath \ldots$. $\mathbb{Z}_{2}$ of the symmetric group $S_{q}$, acts on $\left(S^{n}\right)^{q-1}$ (with antipodal action on an $S^{n}$-factor whenever its children are exchanged, such that the partition $p(x)$ for $x \in X$ stays the same up to permutation of the indices) and on $\operatorname{Conv}\left(S^{n}\right)^{q}$ (as it acts on the leaves). This makes $p$ into a $G$-equivariant map.

We would like to define a test-map

$$
t:\left(S^{n}\right)^{q-1} \longrightarrow S_{n}(M \times \mathbb{R})^{q}
$$

whose $k$ 'th coordinate at $x=\left(x_{1}, \ldots, x_{q-1}\right)$ is given by

$$
\left(f\left(c\left(p_{k}(x)\right)\right), \operatorname{vol}\left(p_{k}(x)\right)\right)
$$

such that the preimage of $\Delta:=\Delta_{(M \times \mathbb{R})^{q}}$ corresponds to the partitions of $S^{n}$ into $q$ convex sets of equal volume and equal $g$-images of their center points. However $c$ is not continuous at some of the convex sets with zero volume. Thus we replace $c$ in $(2)$ by a slightly deformed map $c^{\prime}$ : First, let $\gamma_{C}$ be the shortest geodesic on $S^{n}$ between $\gamma_{C}(0)= \pm x_{1}$ and $\gamma_{C}(1 / 2 q)=c(C)$, where the sign in front of the vector $x_{1}$ (in the sphere corresponding to the root of $T$ ) depends on whether the leaf of $T$ corresponding to the convex set $C$ is on the left or on the right side of the root. If $\operatorname{vol}(C)=0$ then $\gamma_{C}$ might not be defined except for its end point $\gamma_{C}(0)$. We then define

$$
c^{\prime}(C):= \begin{cases}c(C) & \text { if } \operatorname{vol}(C) \geq 1 / 2 q, \\ \gamma_{C}(\operatorname{vol}(C)) & \text { if } \operatorname{vol}(C) \leq 1 / 2 q .\end{cases}
$$

The so defined $t: x \mapsto\left(f\left(c^{\prime}\left(p_{k}(x)\right)\right), \operatorname{vol}\left(p_{k}(x)\right)\right)_{k}$ is indeed continuous and $t^{-1}(\Delta)$ is the set of convex equipartitions of $S^{n}$ such that $g$ maps all centers of the convex parts to the same point in $M$. 
The test-map $t$ factors as

$$
X \stackrel{i}{\longrightarrow} Y^{q} \stackrel{(f \times \mathrm{id})^{q}}{\longrightarrow}(M \times \mathbb{R})^{q}
$$

where $Y:=S^{n} \times \mathbb{R}$.

Lemma 6.2 (An index bound for $\left.\left(S^{n}\right)^{q-1}\right)$. For $G=\mathbb{Z}_{2} \imath \ldots \curlywedge \mathbb{Z}_{2} \subseteq S_{q}$ and $\mathbb{F}_{2}$-coefficients,

$$
e\left(W_{q}\right)^{n} \notin \operatorname{ind}_{G}^{F H}\left(\left(S^{n}\right)^{q-1}\right) .
$$

Proof. Consider the map $m:\left(S^{n}\right)^{q-1} \rightarrow W_{q}^{\oplus n}$ given by

$$
x \mapsto\left(\sum_{N_{i} \in P_{k}} \pm \operatorname{pr}_{S^{n} \rightarrow \mathbb{R}^{n}}\left(x_{i}\right)\right)_{k=1 \ldots q},
$$

where $\operatorname{pr}_{S^{n} \rightarrow \mathbb{R}^{n}}: S^{n} \rightarrow \mathbb{R}^{n}$ is the standard projection to the first $n$ coordinates; for every leaf $L_{k}, P_{k}$ is the set of interior nodes in $T$ that lie on the shortest path from the root $N_{1}$ to $L_{k}, \ell(i)$ is the height of node $i$ in the tree (i.e. the distance to $N_{1}$ ), and the sign at $N_{i} \in P_{k}$ depends on whether the path $P_{k}$ continues at the right or the left subtree at node $N_{i}$.

We have that the sum of all $q \mathbb{R}^{n}$-coordinates of this test-map is zero, since the sum for $P_{k}$ cancels with the sum for the reflected $P_{k}$. Furthermore, $m$ is $G$-equivariant, and $m^{-1}(0)=\{(0, \ldots, 0, \pm 1)\}^{q-1}$ is the set of $(q-1)$-tuples $x$ such that every $x_{i}$ is the north or the south pole of $S^{n}$. These are regular points of $m$, and modulo $G$ this is exactly one preimage.

Remark 6.3 (Odd prime powers). There is an analogous lemma for odd prime powers $q=p^{\ell}$ if $n$ is odd: Here, $G=\mathbb{Z}_{p} \imath \ldots \curlywedge \mathbb{Z}_{p} \subseteq S_{q}, \mathbb{Z}_{p}$ acts on $S^{n}=S^{1} * \ldots * S^{1}$ diagonally, and we use

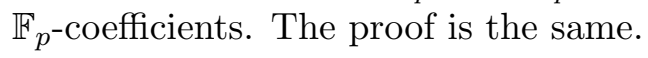

Remark 6.4 (An index bound for configuration spaces). Let $F_{q}\left(\mathbb{R}^{n+1}\right)$ denotes the configuration space of $q$ pairwise distinct points of $\mathbb{R}^{n+1}$. Hung [Hun90, 11$]$ (see also KarasevVolovikov [KV11, 5.2]) constructed an embedding $\left(S^{n}\right)^{q-1} \hookrightarrow_{G} F_{q}\left(\mathbb{R}^{n+1}\right)$ as follows: The first element $x_{1} \in S^{n}$ determines a pair of antipodal points on $\mathbb{R}^{n+1}$. The next two elements $x_{2}, x_{3} \in S^{n}$ are used to split these two antipodal points into four points on $\mathbb{R}^{n+1}$. And so on. Using this embedding, lemma 6.2 provides a simple proof for

$$
e\left(W_{q}\right)^{n} \notin \operatorname{ind}_{G}^{F H}\left(F_{q}\left(\mathbb{R}^{n+1}\right)\right) .
$$

For an application of this index bound on convex partitions see Blagojević-Ziegler [BZ12]. More general index calculations for configuration spaces can be found in Karasev Kar09a. and Blagojević-Lück-Ziegler [BLZ12].

Since we need only the non-vanishing of $e\left(W_{q}\right)^{k}$, we may restrict the configuration space $\left(S^{n}\right)^{q-1}$ to some $G$-invariant subspace $\left(S^{k}\right)^{q-1}$. Here, $G$-invariance means that we can choose the $k$-dimensional equators $S^{k} \subseteq S^{n}$ independently as long as they agree on each height (with respect to $T$ ). Choosing these equators well-distributed enough will assure the $\varepsilon$-neighborhood condition in theorem 6.1 .

Using Volovikov's theorem 1.1 finishes the proof of theorem 6.1 . 
Remark 6.5. Karasev and Volovikov [KV11] observed that when we remove the condition that the $C_{i}$ have to be $\varepsilon$-close to some $k$-dimensional equators of $S^{n}$, then the mass partition theorem 6.1 holds also for odd prime powers: For this they used weighted Voronoi decompositions.

A parametrized version of theorem 6.1 follows analogously using theorem 4.1 . This in turn implies the parametrized waist of the sphere theorem 1.3 using the same analytic part as in Gromov [Gro03].

Acknowledgements. I want to thank Vera Vértesi, Mark Goresky, and Roman Karasev for very useful discussions.

\section{References}

[BBM11] Pavle V. M. Blagojević, Aleksandra Dimitrijević Blagojević, and John McCleary. Spectral sequences in combinatorial geometry: Cheeses, inscribed sets, and Borsuk-Ulam type theorems. Topology Appl., 158(15):1920-1936, 2011.

[BLZ12] Pavle V. M. Blagojević, Wolfgang Lück, and Günter M. Ziegler. Equivariant topology of configuration spaces. arxiv:1207.2852, 2012.

[BMZ11] Pavle V. M. Blagojević, Benjamin Matschke, and Günter M. Ziegler. Optimal bounds for a colorful Tverberg-Vrećica type problem. Adv. Math., 226:5198-5215, 2011.

[Bor33] Karol Borsuk. Drei Sätze über die n-dimensionale euklidische Sphäre. Fund. Math., 20:177190, 1933.

[BZ12] Pavle V. M. Blagojević and Günter M. Ziegler. Convex equipartitions via equivariant obstruction theory. arxiv:1202.5504, 2012.

[CJ09] Michael C. Crabb and Jan W. Jaworowski. Theorems of Kakutani and Dyson revisited. J. Fixed Point Theory Appl., 5(2):227-236, 2009.

[dMdS07] Denise de Mattos and Edivaldo L. dos Santos. A parametrized Borsuk-Ulam theorem for a product of spheres with free $\mathbb{Z}_{p}$-action and free $S^{1}$-action. Algebr. Geom. Topol., 7:17911804, 2007.

[Dol87] Vladimir L. Dol'nikov. Common transversals for families of sets in $\mathbb{R}^{n}$ and connections between theorems of Helly and Borsuk. (in Russian) Dokl. Akad. Nauk USSR, 297(4):777$780,1987$.

[Dol88] Albrecht Dold. Parametrized Borsuk-Ulam theorems. Comment. Math. Helvetici, 63:275$285,1988$.

[Dol92] Vladimir L. Dol'nikov. A generalization of the ham sandwich theorem. Mat. Zametki, 52(2):27-37, 155, 1992. Translation in Math. Notes 52 (1993), no. 1-2, 771-779.

[FH87a] Edward Fadell and Sufian Y. Husseini. Index theory for $G$-bundle pairs with applications to Borsuk-Ulam type theorems for $G$-sphere bundles. In Nonlinear analysis, pages 307-336. World Sci. Publishing, 1987.

[FH87b] Edward Fadell and Sufian Y. Husseini. Relative cohomological index theories. Adv. Math., 64:1-31, 1987.

[FH88] Edward Fadell and Sufian Y. Husseini. An ideal-valued cohomological index theory with applications to Borsuk-Ulam and Bourgin-Yang theorems. Ergodic Theory and Dynamical Systems, 8*:73-85, 1988. 
[FH89] Edward Fadell and Sufian Y. Husseini. Index theory for noncompact group actions with applications to Borsuk-Ulam theorems. In Topological fixed point theory and applications (Tianjin, 1988), Lecture Notes in Math. 1411, pages 52-68. Springer, 1989.

[Gro03] Mikhail L. Gromov. Isoperimetry of waists and concentrations of maps. GAFA, 13:178-215, 2003.

[Hel23] Eduard Helly. Über Mengen konvexer Körper mit gemeinschaftlichen Punkten. Jahresbericht Deutsch. Math. Vereining., 32:175-176, 1923.

[Hun90] Nguyên H. V. Hung. The mod 2 equivariant cohomology algebras of configuration spaces. Pacific J. Math., 143(2):251-286, 1990.

[IJ92] Marek Izydorek and Jan W. Jaworowski. Parametrized Borsuk-Ulam theorems for multivalued maps. Proc. Amer. Math. Soc., 116(1):273-278, 1992.

[IR92] Marek Izydorek and Sławomir Rybicki. On parametrized Borsuk-Ulam theorem for free $Z_{p^{-}}$ action. In Algebraic topology (San Feliu de Guíxols, 1990), Lecture Notes in Math. 1509, pages 227-234. Springer, 1992.

[Jaw81a] Jan W. Jaworowski. A continuous version of the Borsuk-Ulam theorem. Proc. Amer. Math. Soc., 82(1):112-114, 1981.

[Jaw81b] Jan W. Jaworowski. Fibre-preserving maps of sphere-bundles into vector space bundles. In Fixed point theory (Sherbrooke, Que., 1980), Lecture Notes in Math. 886, pages 154-162. Springer, 1981.

[Jaw04] Jan W. Jaworowski. Bundles with periodic maps and mod $p$ Chern polynomial. Proc. Amer. Math. Soc., 132(4):1223-1228, 2004.

[Kar07] Roman N. Karasev. Tverberg's transversal conjecture and analogues of nonembeddability theorems for transversals. Discrete Comput. Geom., 38:513-525, 2007.

[Kar09a] Roman N. Karasev. The genus and the category of configuration spaces. Topology Appl., 156(14):2406-2415, 2009.

[Kar09b] Roman N. Karasev. Theorems of Borsuk-Ulam type for planes, and flat transversals of families of convex compact sets. Rossǐ̌skaya Akademiya Nauk. Matematicheskiน Sbornik, 200(10):39-58, 2009. Translation in Sb. Math. 200 (2009), no. 9-10, 1453-1471.

[KM96] Basil Shaju Koikara and Himadri Kumar Mukerjee. A Borsuk-Ulam theorem for maps of fibre bundles with manifolds as fibres. Arch. Math. (Basel), 66(6):499-501, 1996.

[KV11] Roman N. Karasev and Aleksei Yu. Volovikov. Waist of the sphere for maps to manifolds. arXiv:1102.0647, 2011.

[Mat02] Jiř́ Matoušek. Lectures on Discrete Geometry, volume 212 of Graduate Texts in Math. Springer, 2002.

[Mat11] Benjamin Matschke. Equivariant topology methods in discrete geometry. PhD thesis, Freie Universität Berlin, 2011.

[Mat12] Benjamin Matschke. Successive spectral sequences. In preparation, 2012.

[Mem09] Yashar Memarian. On Gromov's waist of the sphere theorem. arxiv:0911.3972, 2009.

[MK95] Neža Mramor-Kosta. A parametrized Borsuk-Ulam theorem for Banach bundles. Glasnik Matematički. Serija III, 30(50)(1):111-127, 1995.

[MK11] Luis Montejano and Roman N. Karasev. Topological transversals to a family of convex sets. Discrete Comput. Geom., 46(2):283-300, 2011. 
[Nak61] Minoru Nakaoka. Homology of the infinite symmetric group. Ann. Math., 73:229-257, 1961.

[Nak84] Minoru Nakaoka. Equivariant point theorem for fibre-preserving maps. Osaka J. Math., 21:809-815, 1984.

[Nak89] Minoru Nakaoka. Parametrized Borsuk-Ulam theorems and characteristic polynomials, pages 155-170. Topological fixed point theory and applications, Lecture Notes in Math. 1411. Springer, 1989.

[Sin11] Mahender Singh. Parametrized Borsuk-Ulam problem for projective space bundles. Fundamenta Mathematicae, 211(2):135-147, 2011.

[SSST11] Thomas Schick, Robert Samuel Simon, Stanislaw Spiecż, and Henryk Toruńczyk. A parameterized version of the Borsuk-Ulam. arxiv:0709.1774, 2011. To appear in Bull. London Math. Soc.

[tD87] Tammo tom Dieck. Transformation Groups, volume 9 of Studies in Math. de Gruyter, 1987.

[TV93] Helge Tverberg and Siniša Vrećica. On generalizations of Radon's theorem and the ham sandwich theorem. Europ. J. Combinatorics, 14:259-264, 1993.

[Tve66] Helge Tverberg. A generalization of Radon's theorem. J. Lond. Math. Soc., 41:123-128, 1966.

[Tve81] Helge Tverberg. A generalization of Radon's theorem II. Bull. Austral. Math. Soc., 24:321$325,1981$.

[Vol80] Aleksei Yu. Volovikov. On the Bourgin-Yang theorem. Russian Math Surveys, 35(3):196200, 1980 .

[Vol83] Aleksei Yu. Volovikov. Mappings of free $\mathbb{Z}_{p}$-spaces into manifolds. Math. USSR Izv., 20(1):35-54, 1983.

[Vol92] Aleksei Yu. Volovikov. A theorem of Bourgin-Yang type for $\mathbb{Z}_{p}^{n}$-action. Matematicheskii Sbornik, 183(7):115-144, 1992. Translation in Russian Acad. Sci. Sb. Math. 76 (1993), no. $2,361-387$.

[Vol96] Aleksei Yu. Volovikov. On fiberwise G-mappings. Uspekhi Mat. Nauk, 51(3(309)):189-190, 1996. Translation in Russian Math. Surveys 51 (1996), no. 3, 575-577.

[Vre03] Siniša Vrećica. Tverberg's conjecture. Discrete Comput. Geometry, 29:505-510, 2003. arxiv:0207011.

[Živ96] Rade T. Živaljević. User's guide to equivariant methods in combinatorics. Publ. Inst. Math. Belgrade, 59(73):114-130, 1996.

[Živ98] Rade T. Živaljević. User's guide to equivariant methods in combinatorics II. Publ. Inst. Math. Belgrade, 64(78):107-132, 1998.

[Živ99] Rade T. Živaljević. The Tverberg-Vrećica problem and the combinatorial geometry on vector bundles. Israel J. Math., 111:53-76, 1999.

[ŽV90] Rade T. Živaljević and Siniša Vrećica. An extension of the ham sandwich theorem. Bull. Lond. Math. Soc., 22:183-186, 1990.

Benjamin Matschke

Forschungsinstitut für Mathematik, ETH Zürich

benjaminmatschke@math.ethz.ch 